\title{
Escarabajos (Coleoptera: Scarabaeoidea) del departamento del Chocó, Colombia
}

\section{Beetles (Coleoptera: Scarabaeoidea) from Department of Chocó, Colombia}

\author{
Jhon César Neita-Moreno*
}

\begin{abstract}
Resumen
Se revisaron 12.654 especímenes de la superfamilia Scarabaeoidea (Insecta: Coleoptera) depositadas en nueve colecciones colombianas y una del extranjero. Se identificaron un total de 183 especies, distribuidas en 74 géneros y seis familias. Se reportan por primera vez para Colombia las especies Cyclocephala almitana Dechambre, C. brevis Höhne, C. krombeini Endrödi, C. ovolum Bates, C. santaritae Ratcliffe, C. vidanoi Dechambre, Homophileurus tricuspis Prell, Phileurus youngi Ratcliffe (Dynastinae); Chlorota jamesonae Soula, Pelidnota hirsuta Ohaus, P. paralella Hardy y $P$. punctulata Bates (Rutelinae) y Amithao cavifrons (Burmeister) (Cetoniinae).
\end{abstract}

Palabras clave: Superfamilia; Distribución; Taxonomía; Chocó.

\begin{abstract}
A total of 12,654 specimens of Scarabaeoidea (Insecta: Coleoptera) were revised and are deposited in 9 collections in Colombian and one foreign ones. A total of 183 species, distributed in 74 genera and six subfamilies were identified. Cyclocephala almitana Dechambre, C. brevis Höhne, C. krombeini Endrödi, C. ovolum Bates, C. santaritae Ratcliffe, C. vidanoi Dechambre, Homophileurus tricuspis Prell, Phileurus youngi Ratcliffe (Dynastinae); Chlorota jamesonae Soula, Pelidnota hirsuta Ohaus, $P$. paralella Hardy y $P$. punctulata Bates (Rutelinae) y Amithao cavifrons (Burmeister) (Cetoniinae) are record for Colombia for the first time.
\end{abstract}

Keywords: Superfamily; Distribution; Taxonomy; Chocó.

\section{Introducción}

Los escarabajos escarabaeidos comprenden uno de los grupos más especiosos en el orden Coleoptera. Estos escarabajos son parte de una fauna conspicua en las regiones tropicales del mundo, sobre todo en el Neotrópico. En el Chocó, dadas sus características y ubicación geográfica, este grupo presenta una diversidad muy importante. El tamaño, las formas, los colores y su elaborada cornamenta en los machos, así como su historia natural hacen de este grupo de escarabajos animales fascinantes y de interés para los coleccionistas, amantes de las colecciones y naturistas. Dentro de esta familia de escarabajos encontramos a los famosos escarabajos Goliat de Áfri- ca (Goliathus goliathus (L.)), escarabajos rinocerontes (Megasomas elephas (L.)) y el escarabajo Hércules (Dynastes hercules (L.)) de América Tropical con más de $150 \mathrm{~mm}$. Este grupo de organismos incluye aproximadamente 35.000 especies (Neita y Gaigl 2008).

Los adultos de los escarabaeidos, se distinguen por las siguientes características: protórax abultado con coxas largas y tibias usualmente dentadas con una sola espina; alas posteriores con reducción de la venación; antenas lameladas; placas coxales anteriores ausentes; el segundo esternito abdominal representado por una sola porción lateral; el octavo segmento abdominal formando un verdadero pigidio y no cancelado por el séptimo segmento; cuatro tu-

* Universidad Nacional de la Plata, Laboratorio de Sistemática y Biología Evolutiva, La Plata, Argentina.

e-mail: cneitaj@unal.edu.co

Fecha recepción: Noviembre 23, 2010

Fecha aprobación: Diciembre 20, 2010 
bos de malpigi (Scholtz 1990).

Las larvas son blancas, cabeza bien esclerosada, cuerpo usualmente en forma de «C» (larva con la forma típica escarabaeiforme), antenas bien desarrolladas, dos a tres pares de patas, no hay urogonfus y los espiráculos son usualmente cribiformes.

A pesar de ser los Scarabaeoideos una de las superfamilias más diversas dentro del orden Coleoptera, su conocimiento en ecosistemas tan importantes y estratégicos como el Chocó es incipiente. Basado en lo anterior, este trabajo tiene como objetivo identificar a través de un inventario la diversidad del grupo Scarabaeoidea del departamento del Chocó.

\section{Materiales y métodos}

Para llevar a cabo este trabajo se revisaron las colecciones entomológicas del país con el fin de identificar el material depositado en ellas procedentes del departamento del Chocó. En total se revisaron nueve colecciones en el país y una colección en el exterior.

MUSENUEV Museo Entomológico, Universidad del Valle, Cali, Valle del Cauca.

MEUC Museo de Entomología, Universidad de Cundinamarca, Fusagasugá, Cundinamarca.

UNAB Museo Entomológico, Facultad de Agronomía, Universidad Nacional de Colombia, Bogotá, DC, Cundinamarca.

ICN-MHN Colección de Zoología, Instituto de Ciencias Naturales, Universidad Nacional de Colombia, Bogotá DC.

MPUJ Museo Javeriano de Historia Natural «Lorenzo Uribe», Pontificia Universidad Javeriana, Bogotá, D.C, Cundinamarca.

$\mathrm{IAvH}$ Colecciones Biológicas, Sección de Entomología, Instituto de Investigaciones Alexander von Humboldt, Villa de Leyva, Boyacá.

MEFLG Museo Entomológico Francisco Luis Gallego, Universidad Nacional de Colombia, sede Medellín, Antioquia.

MUA Colección de Ciencias Naturales,
Universidad de Antioquia, Medellín, Antioquia.

CJCN Colección Jhon César Neita, Bogotá DC, Cundinamarca.

IEXA Instituto de Ecología de México, Xalapa,Veracruz-Colección Passalidae (Dr. Pedro Reyes).

\section{Resultados y discusión}

En total se revisaron 12.654 especímenes depositados en estas colecciones. Se identificaron un total de 183 especies distribuidas en seis familias, diez subfamilias y 74 géneros (Apéndice I).

La familia mejor representada fue Scarabaeidae «Pleurosticti» con el 68,3\% de las especies, seguida por Scarabaeidae «Laparosticti» con el $21.9 \%$, Passalidae con el 7.7\%, Geotrupidae con el 1.1\%, Trogidae e Hybosoridae con el $0.5 \%$ cada uno (Figura 1, Apéndice II).

Familia Scarabaeidae «Pleurosticti». En esta familia se identificaron 125 especies, agrupadas en cuatro subfamilias y 15 tribus. La subfamilia mejor representada es Dynastinae con 48\%, seguida por Rutelinae 33.3\%, Cetoniinae 12.2\% y Melolonthinae con el $6.5 \%$.

La subfamilia Dynastinae estuvo representada por el 100\% de las tribus reportadas para el Neotrópico, seis en total. La tribu mejor representada fue Cyclocephalini con $64.3 \%$ de las especies, seguida de Oryctini $10.7 \%$, Pentodontini y Phileurini con el $8.9 \%$, Dynastini con 5.4\% y Agaocephalini con el $1.8 \%$ de las especies.

Tribu Cyclocephalini. En esta tribu se encontraron 36 especies, $69.4 \%$ del género Cyclocephala Dejean, seguido de Aspidolea Burmeister con 11.1\%, Ancognatha Erichson y Dyscinetus Harold con $5.6 \%$, y Erioscélis Burmeister, Mimeoma Casey y Stenocrates Burmeister con 2.8\% de las especies.

Dentro de la tribu se informaron por primera vez para Colombia las especies: Cyclocephala almitana, C. brevis, C. brevippenis, C. krombeini, C. ovolum, C. santaritae, C. vidanoi.

La segunda tribu mejor representada fue Oryctini. Se hallaron ocho especies de las cuales $37.5 \%$ pertenece al género Strategus Kirby. Los géneros Coelosis Hope, Enema Hope y Podischnus Burmeister con $12.5 \%$ cada una. 


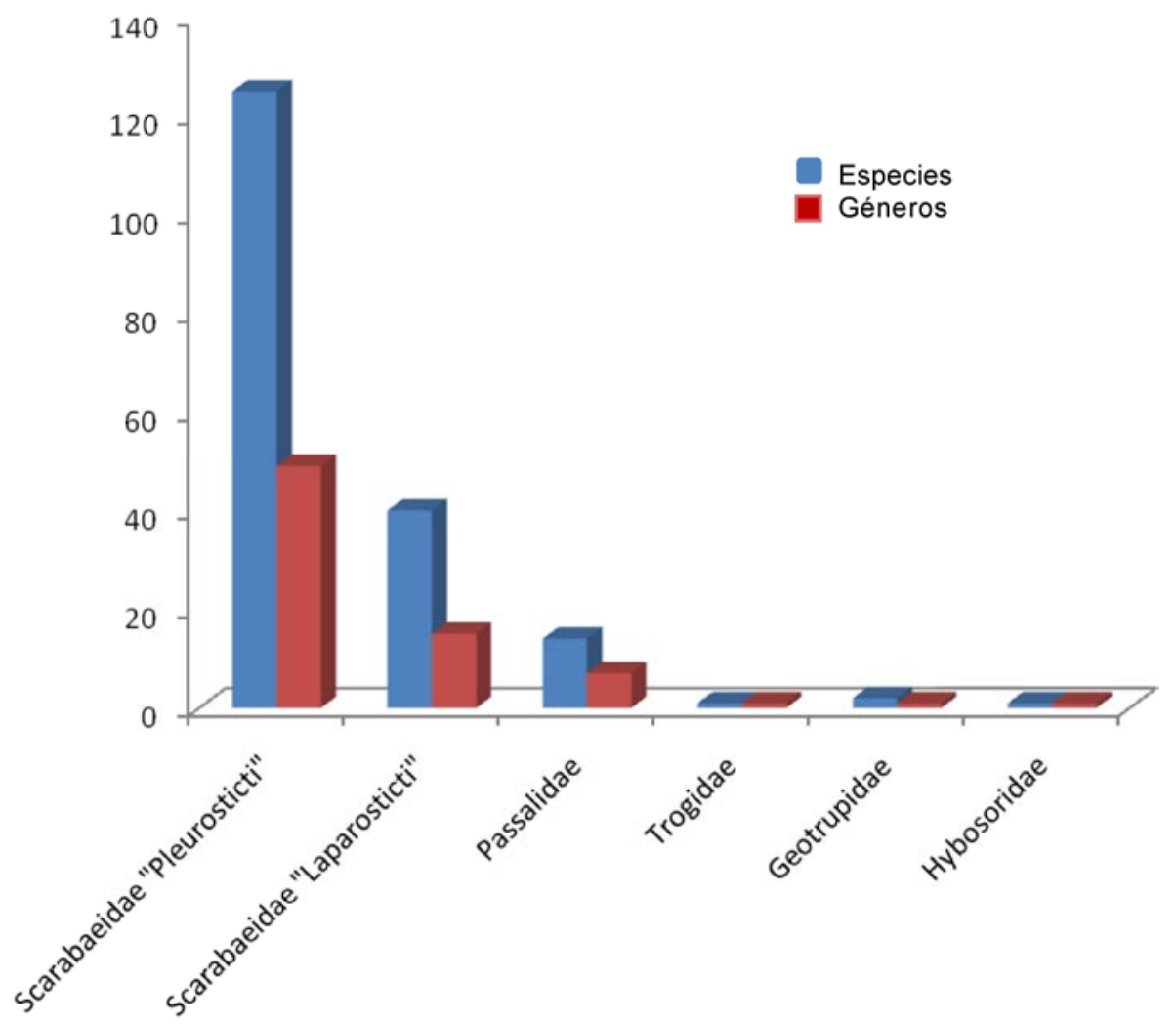

Figura 1. Composición de la superfamilia Scarabaeoidea en el departamento del Chocó.

Tribu Pentodontini. En esta tribu se informan seis especies, distribuidas en cuatro géneros. El género mejor representado es Tomarus Erichson con tres especies; los demás géneros, es decir, Euetheola Bates, Parapucaya Prell y Pucaya Ohaus con $16.7 \%$ de las especies cada una.

Tribu Phileurini. Sólo tres géneros han sido capturados; Homophileurus Kolbe y Phileurus Latreille con $40 \%$ cada uno. El género Hemiphileurus Kolbe con $20 \%$ del total de las especies con Hemiphileurus elbitae (Neita y Ratcliffe 2010).

Las dos especies del género Homophileurus: $H$. tricuspis y el género Phileurus: $P$. youngi son nuevo reporte para Colombia.

Tribu Dynastini. Se capturaron dos géneros: Megasoma Kirby con $66.7 \%$ de las especies y Dynastes MacLeay con $33.3 \%$.

Por último, la tribu Agaocephalini fue la menos rica en especies con sólo una especie del género Spodistes Hope, aún sin identificar.

La subfamilia Rutelinae estuvo representada por las cinco tribus presentes en el nuevo mundo. La tribu con mayor riqueza de especies es Rutelini
MacLeay con $54.8 \%$ de las especies, seguida de Anomalini Samouelle con 28.6\%, Geniatini Ohaus con 9.5\%, Anatistini Brême con 4.8\% y Anoplognathini Ohaus con $2.4 \%$ del total de las especies.

En la tribu Rutelini se informan 23 especies, siendo el género Pelidnota MacLeay el mejor representado con $30.4 \%$ de las especies, seguido de Rutela Latreille con $13 \%$, de los géneros Chlorota Burmeister, Dorystethus Banchard, Lagochile Hoffmannsegg y Macraspis MacLeay con 8.8\% cada uno y los demás géneros Chrysina Kirby, Chrysophora Serville, Cnemida Kirby, Microrutela Jameson y Ptenomela Bates con $4.3 \%$ de las especies.

Dentro de la tribu son nuevos reportes para Colombia Chlorota jamesonae Soula, Pelidnota hirsuta Ohaus, $P$. paralella Hardy y $P$. punctulata Bates.

En la tribu Anomalini se informan 12 especies, distribuidas en dos géneros: Paranomala Casey y Strigoderma Burmeister.

Para el género Paranomala se identificaron sólo cinco especies de las diez totales pero aún no hay 
trabajo para este género a nivel de América; este es el género más grande dentro de la superfamilia Scarabaeoidea con un total aproximado de 1.000 especies de amplia distribución a nivel mundial. Por su parte, el género Strigoderma presentó dos especies con todos los especímenes hembra lo cual dificultó el nivel de identificación específico.

La tribu Geniatini está representada por cuatro especies y dos géneros, Geniates Kyrbi y Leucothyreus MacLeay, cada uno con dos especies.

Por último, la tribu Anoplognatini estuvo representada por una sola especie: Platycoelia occidentalis Ohaus, típica de la zona alta andina de la cordillera Occidental, siendo éste el único reporte de distribución de la especie en Colombia, lo que hace suponer que la especie es endémica de esta zona con su especie hermana Platycoelia valida Burmeister en la cordillera Oriental, en los valles del río Magdalena de los departamentos de Cundinamarca y Antioquia.

La subfamilia Cetoniinae Leach. Se registran 15 especies, distribuidas en siete géneros y dos tribus. La tribu mejor representada fue Gymnetini con 93.3\% de las especies y $85.7 \%$ de los géneros. Por su parte, la tribu Cetoniini con una sola especie, representa $6.6 \%$ de las especies y $14.3 \%$ de los géneros. En la subfamilia se informa por primera vez para Colombia y Sudamérica la especie Amithao cavifrons (Burmeister 1842).

Por último, la subfamilia con menor riqueza de especies es la Melolonthinae con ocho especies en total, distribuidas en cinco géneros y dos tribus. La tribu mejor representada fue Melolonthini con un único género, Phyllophaga Harris, con $62.5 \%$ del total de las especies. Por su parte, la tribu Macrodactylini con $37.5 \%$ estuvo representada por los géneros Plectris Serville con dos especies, Baribas Blanchard, Ceraspis Serville e Isonychus Mannerheim con una especie cada una.

Familia Scarabaeidae «Laparosticti». En esta familia se identificaron 38 especies, agrupadas en una subfamilia Scarabaeinae y cinco tribus. La tribu mejor representada es Canthonini con 36.8\%, seguida de Dichotomini $28.9 \%$, Phanaeini con $21.1 \%$, y por último Onthophagini con $7.9 \%$ y Eurysternini con $5.3 \%$.La tribu mejor representada fue Canthonini. Se encontraron 14 especies de las cuales $35.7 \%$ perte- nece al género Deltochilum Eschsoltz, seguida de Dichotomius Hope con $28.6 \%$, y de Canthon Hoffmannsegg 21.4\%; los demás géneros Cryptocanthon Bathasar y Malagoniella Martínez presentaron $7.1 \%$ cada uno.

La segunda tribu mejor representada es Dichotomini. En esta tribu se hallaron 11 especies, de las cuales $72.7 \%$ pertenece al género Canthidium Erichson, seguido de Bdelyrus Harold, Ontherus Erichson y Uroxys Westwood con 9.1\% de las especies cada uno.

La tribu Phanaeini. En esta tribu se identifican ocho especies, distribuidas en cuatro géneros. El género mejor representado es Coprophanaeus Olsoufieff con $50 \%$ de las especies, seguido de Oxysternon Laporte-Castenau con 25\%; los demás géneros, es decir, Phanaeus MacLeay y Sulcophanaeus Olsoufieff, cada uno con $12.5 \%$ de las especies. Las tribus Eurysternini con dos especies y un género Eurysternon Dalman.

Por último, la tribu Onthophagini con tres especies y un género Onthophagus Latreille.

Familia Passalidae. La familia Passalidae está representada por 14 especies, siete géneros y dos subfamilias; la subfamilia Passalinae muestra el mayor número de especies, con $57.1 \%$ y Proculinae con $42.9 \%$. No obstante, la subfamilia Proculinae informa la mayor cantidad de géneros con $71.4 \%$ y Passalinae $28.6 \%$ del total. La subfamilia Passalinae está representada por los géneros Passalus Fabricius con $87.5 \%$ de las especies y Paxillus MacLeay con $12.5 \%$.

Por su parte, en la subfamilia Proculinae se identificaron cinco géneros, de los cuales Verres Kaup representa $33.3 \%$ de las especies. Los otros géneros, es decir, Odontotaenius Kuwert, Petrejoides Kuwerrt, Pseudoarrox Reyes-Castillo y Veturius Kaup tienen $16.7 \%$ cada uno.

Familia Geotrupidae. La familia Geotrupidae está representada por la subfamilia Athyreinae con la especie Neoathyreus lanei (Martínez).

Familia Hybosoridae. Está familia está representada por dos especies en dos géneros y dos subfamilias. La subfamilia Anaidinae con la especie Anaides aff. planus Ocampo 2006 y Ceratocanthinae con una especie Germarostes sp.

Familia Trogidae. Esta familia está representada por una especie del género Omorgus sp. 
Tabla 1. Trabajos realizados donde se citan algunas especies de Scarabaeoidea para el departamento del Chocó

\begin{tabular}{lcc}
\hline & Géneros & Especies \\
\hline $\begin{array}{l}\text { Medina et al. 2001 a } \\
\text { Reyes-Castillo \& }\end{array}$ & 13 & 24 \\
Amat-Garcia 2001 & 5 & 11 \\
Restrepo et al. 2003 & 8 & 17 \\
Amat et al. 2004 & 2 & 3 \\
Amat-Garcia \& & 32 & 92 \\
Trujillo 2005 & 13 & 23 \\
Neita et al. 2003 & 38 & 65 \\
Neita et al. 2006 & & \\
\hline
\end{tabular}

a. Las especies Canthidium centrale (Bouncomont 1928), Canthidium decoratum (Perty 1830), Deltochilum parile (Bates 1887) son listadas con una incógnita.

b. La especie Ancognatha ustulata (Burmeister 1847) es una especie de los bosques alto-andinos de la cordillera Oriental, por lo que es posible que este reporte sea errado.

\section{Discusión}

En Colombia son pocos los trabajos que se han hecho sobre el conocimiento de este grupo de organismos. Los trabajos realizados en torno al conocimiento de la superfamilia hasta el momento son los siguientes (Tabla 1).

Como se puede observar, son pocos los informes de este grupo de organismos para el Chocó. El conocimiento de la fauna aún es muy incipiente. De las ocho formaciones vegetales presentes en el departamento tan sólo una, la selva pluvial central o bosque pluvial tropical (bp-T) es moderadamente conocida en su parte baja. No obstante, con mayor grado de altitud dentro de ella, ya sea en el piedemonte occidental de la cordillera Occidental o el piedemonte oriental de la serranía del Baudó, se puede aumentar la riqueza del grupo en este ecosistema.

Es posible que muestreos más intensivos en otras formaciones vegetales impliquen no sólo aumento en la riqueza y diversidad del grupo para el departamento, sino también la posibilidad de conseguir nuevas taxas para la ciencia.

La riqueza del grupo Scarabaeidae «Laparosticti» es muy baja en la selva pluvial central baja si se compara con otros grupos como «Pleurosticti» o escarabajos fitófagos. La disponibilidad de recurso y presencia de una gran variedad de microhábitats dentro de las sucesiones vegetales en este ecosistema provee las condiciones necesarias para una mayor ri- queza del grupo. Por su parte, los coprófagos o grupo «Laparosticti» presentan una muy baja riqueza en estas selvas en comparación con los reportados para otros sitios del Chocó biogeográfico (Howden \& Young 1981) en Panamá. Hay varias hipótesis que soportan esta teoría con respecto a la baja riqueza del grupo; la primera y la más fuerte es la ausencia de la megafauna herbívora presente en otras zonas como el norte del Chocó, lo que aporta una mayor cantidad de recursos para estas especies de escarabajos.

Los otros grupos como Passalidae, Trogidae, Geotrupidae e Hybosoridae presentan una baja diversidad más por su falta de muestreo, reflejado en la ausencia de ejemplares en los museos de Colombia, que por baja diversidad del grupo en la zona. No obstante, es posible que Passalidae presente una muy buena diversidad, porque la presencia de troncos en descomposición en los diferentes hábitats favoreciendo así el establecimiento de familias y con ello mayor cantidad de especies.

Futuros trabajos de investigación, tendientes a conocer la diversidad de estos grupos a través de muestreos sistemáticos, contribuirán a un aumento sustancial de la diversidad de estos organismos en todas las formaciones vegetales y muy probablemente se llegarán a encontrar muchas especies endémicas.

Distribución de las especies. El $80 \%$ de las especies presenta una distribución amplia en las Américas. Algunas tienen como sitio más norteño o más sureño el Chocó. Así, se tienen especies como Chrysina argenteola, Chrysophora chrysochlora, Pelidnota chiriquina, Pelidnota parallela, Spodochlamys sp. (Rutelinae), Cyclocephala almitana, Cyclocephala brevippenis, Cyclocephala discolor, Cyclocephala hiekei, Cyclocephala krombeini, Cyclocephala quadripunctata, Cyclocephala vidanoi, Tomarus simillus, Strategus hipposederus, Phileurus youngi (Dynastinae), Amithao cavifrons, Amithao niveosparsus, Gymnetis vandepolli, Guatemalica hueti, Marmarina maculosa (Cetoniinae) Scarabaeidae «Pleurosticti».

Por su parte, en los Scarabaeidae «Laparosticti» se identifican las especies: Bdelyrus seminudus, Canthidium aurifex, Canthidium elegantulum, Canthon aequinoctialis, Canthon moniliatus, Deltochilum loperae, Deltochilum psedoparile, 
Dichotomius gamboaensis, Dichotomius horridus, Ontherus didymus, Onthophagus acuminatus, Phanaeus pyrois, Sulcophanaeus noctis y Uroxys bidentis.

En la familia Passalidae se registran las especies: Odontotaenius striatopunctatus, Passalus (Mitrrhinus) arrowi, Passalus (Passalus) alticola, Passalus (Passalus) interstitialis, Passalus (Passalus) punctiger, Passalus (Passalus) spinulosus, Passalus (Pertinax) incertus, Petrejoides subrecticornis, Veturius platyrhinus.

En las otras familias Trogidae, Geotrupidae e Hybosoridae las especies son de amplia distribución en la región Neotropical.

Así y con base en este estudio, se ratifica la importancia del Chocó en la biogeografía de este grupo de organismos, pues se convierte en la zona de transición de las faunas de Centro América y Sur América.

\section{Literatura citada}

Amat-García GD, Blanco-Vargas E, Reyes-Castillo P. 2004. Lista de especies de los escarabajos pasálidos (Coleoptera: Passalidae) de Colombia. Biota Colomb. 5(2): 173-82.

Amat G, Trujillo D. 2005. Escarabajos (Coleoptera: Scarabaeidae) en el Chocó biogeográfico. p. 745-54. En: Rangel O. (ed). Colombia Diversidad Biotica IV. Bogotá: Universidad
Nacional de Colombia, Conservación Internacional de Colombia.

Medina CA, Lopera A, Vitolo A, Gill B. 2001. Escarabajos coprófagos (Coleoptera: Scarabaeidae: Scarabaeinae) de Colombia. Biota Colomb. 2 (2): 131-44.

Neita-Moreno J, Pardo-Locarno L, Quinto-Murray D, Cuesta N. 2003. Los escarabajos copronecrófagos (Coleoptera: Scarabaeidae) en la Parcela Permanente de Investigación de Biodiversidad, Corregimiento de Salero-Unión Panamericana. En: Diversidad Biológica de un bosque pluvial tropical (bp-T). Bogotá: Guadalupe Ltda. p. 7990.

Neita-Moreno J, Orozco-Araujo J, Brett C. R. 2006. Escarabajos (Coleoptera: Scarabaeidae «Pleurosticti») de la selva baja del bosque pluvial tropical, Chocó, Colombia. Acta Zool $\operatorname{Mex}(n s) .22$ (2): 1-32.

Neita-Moreno JC, Gaigl. A. 2008. Escarabajos de importancia agrícola en Colombia (Coleoptera: Scarabaeidae «Pleurosticti»). Bogotá: Universidad Nacional de Colombia, Produmedios; $161 \mathrm{pp}$.

Restrepo H, Morón MA, Vallejo F, Pardo L, López A. 2003 Catálogo de Coleoptera: Melolonthidae (Scarabaeidae: Pleurosticti) en Colombia. Folia Entomol Mex. 42 (2): 239-63.

Reyes-Castillo P, Amat G. 2001. Passalidae (Coleoptera) de Colombia. En: Onore G, Reyes-Castillo P, Zunino M. (eds.). Escarabaeidos de Latinoamerica. Estado actual de su conocimiento. Monografía. Madrid: Sociedad Entomológica Aragonesa. p. 35-50.

Scholtz CH. 1990. Phylogenetic trends in the Scarabaeoidea (Coleoptera). J Nat Hist. 24: 1027-66.

\section{Familia Scarabaeidae «Pleurosticti»}

\section{Melolonthinae}

\section{Melolonthini}

Phyllophaga sp.1

Phyllophaga sp.2

Phyllophaga sp.3

Macrodactylini

Baribas sp.

Ceraspis sp.

Isonychus $\mathrm{sp}$.

Plectris sp. 1

Plectris sp.2

\section{Rutelinae}

\author{
Anomalini \\ Paranomala cuacana (Ohaus, 1837) \\ Paranomala cincta (Say, 1835) \\ Paranomala cupricollis (Chevrolat, 1834) \\ Paranomala medellina (Ohaus, 1897) \\ Paranomala undulata (Melsheimer, 1844)
}

\section{Apéndice I}

Paranomala sp.1
Paranomala sp.2
Paranomala sp.3
Paranomala sp.4
Paranomala sp.5
Strigoderma sp.1
Strigoderma sp.2

Rutelini

Chlorota jamesonae Soula, 2002

Chlorota sp.1

Chrysina argenteola (Bates, 1888)

Chrysophora chrysochlora (Latreille, 1811)

Cnemida retusa (Fabricius, 1801)

Dorystethus maximus Ohaus, 1905

Dorystethus sp.

Lagochile sp.1

Lagochile sp.2

Macraspis lucida (Linnaeus, 1767)

Macraspis sp.

Microrutela viridiaurata (Bates, 1888)

Pelidnota chiriquina Bates, 1904

Pelidnota santidonomini (Ohaus, 1905) 
Pelidnota notata Blanchard, 1850

Pelidnota parallela Hardy, 1975

Pelidnota polita (Latreille, 1811)

Pelidnota prolixa Sharp, 1877

Pelidnota punctulata Bates, 1888

Ptenomela poncheli Soula, 2002

Rutela heraldica Perty, 1832

Rutela histrio Sahlberg, 1823

Rutela sanguinolenta Waterhouse, 1874

\section{Anoplognathini}

Platycoelia occidentalis Burmeister, 1844

\section{Geniatini}

Geniates sp. 1

Geniates sp.2

Leucothyreus femoratus Burmeister, 1844

Leucothyreus sp.

\section{Spodochlamyini}

Spodochlamys sp.

Chalcochlamys dorhni Ohaus, 1905

\section{Dynastinae}

\section{Cyclocephalini}

Ancognatha veliae Pardo, González y Montoya, 2006

Ancognatha vulgaris Arrow, 1911

Aspidolea fuliginea Burmeister, 1847

Aspidolea kuntzeni Höhne, 1922

Aspidolea notaticollis Höhne, 1922a

Aspidolea singularis Bates, 1888

Cyclocephala amazona (Linnaeus, 1767)

Cyclocephala almitana Dechambre, 1992

Cyclocephala amblyopsis Bates, 1888

Cyclocephala atripes Bates, 1888

Cyclocephala brevis Höhne, 1923

Cyclocephala brittoni Endrödi, 1964

Cyclocephala carabonaria Arrow, 1911

Cyclocephala discolor (Herbst, 1790)

Cyclocephala fasciolata Bates, 1888

Cyclocephala fulgurata Burmeister, 1847

Cyclocephala gravis Bates, 1888

Cyclocephala hiekei Endrödi, 1975

Cyclocephala krombeini Endrödi, 1979

Cyclocephala ligirina Bates, 1888

Cyclocephala lunulata Burmeister, 1847

Cyclocephala macrophylla Erichson, 1847

Cyclocephala maffafa Burmeister, 1847

Cyclocephala melanocephala (Fabricius, 1775)

Cyclocephala ovolum Bates, 1888

Cyclocephala pardolocarnoi Dechambre, 1995

Cyclocephala prolongata Arrow, 1902

Cyclocephala quadripunctata Höhne, 1923b

Cyclocephala santaritae Ratcliffe, 1992

Cyclocephala stictica Burmeister, 1847

Cyclocephala tutilina Burmeister, 1847

Cyclocephala vidanoi Dechambre, 1996
Dyscinetus dubius (Olivier, 1789)

Dyscinetus dytiscoides Arrow, 1911

Erioscelis columbica Endrödi, 1966

Mimeoma acuta Arrow, 1902

Stenocrates bicarinatus Robinson, 1947

Pentodontini

Euetheola bidentata (Burmeister, 1847)

Parapucaya mazonica Prell, 1912

Pucaya castanea Ohaus, 1910

Tomarus ebenus (De Geer, 1774)

Tomarus fossor (Latreille, 1813)

Tomarus maternus (Prell, 1937)

Tomarus simillus (Endrödi, 1966)

\section{Oryctini}

Coelosis biloba (Linnaeus, 1767)

Enema pan (Fabricius, 1775)

Heterogomphus schoenherri Burmeister, 1847

Megaceras morpheus Burmeister, 1847

Podischnus agenor (Olivier, 1860)

Strategus aloeus (Linnaeus, 1767)

Strategus jugurta Burmeister, 1847

Strategus hipposederusRatcliffe, 1976

\section{Phileurini}

Hemiphileurus elbitaeNeita y Ratcliffe, 2010

Homophileurus quadripuntactus (Beauve et Palisot, 1835)

Homophileurus tricuspis Prell, 1914

Phileurus didymus (Linnaeus, 1767)

Phileurus youngi Ratcliffe, 1992

Agaocephalini

Spodistes grandis Sternberg, 1903

\section{Dynastini}

Dynastes hercules (Linnaeus, 1767)

Megosoma acteon (Linnaeus, 1767)

Megasoma elephas (Fabricius, 1767)

\section{Cetoniinae}

\section{Gymnetini}

Amithao cavifrons (Burmeister, 1844)

Amithao decemguttatum (Waterhouse, 1876)

Amithao niveosparsus Moser, 1913

Cotinis lebasi (Gory \& Percheron, 1833)

Gymnetis coturnix (Burmeister, 1842)

Gymnetis holosericea (Voet, 1779)

Gymnetis pardalis (Gory \& Percheron, 1833)

Gymnetis stellata (Latreille, 1833)

Gymnetis vandepolli (Bates, 1887)

Gymentis wallastoni (Schaum, 1848)

Guatemalica hueti (Chevrolat, 1870)

Hoplopyga liturata (Olivier, 1789)

Marmarina maculosa (Olivier, 1789) 
Cetoniini

Euphoria steiheili Janson, 1878

Familia Scarabaeidae «Laparosticti». Scarabaeinae Latreille, 1802

Dichotomini Halffter \& Matthews, 1966

Bdelyrus seminudus (Bates, 1887)

Canthidium aurifex Bates, 1887

Canthidium centrale Bouncomont, 1928

Canthidium decoratum (Perty, 1830)

Canthidium elegantulum Balthasar, 1939

Canthidium haroldi Preudhomme, 1886

Canthidium macroculare Howden \& Gull, 1987

Canthidium perceptibile Howden \& Young, 1981

Canthidium stenheili Harold, 1880

Ontherus didymus Erichson, 1847

Ontherus trituberculatum Balthasar, 1938

Uroxys bidentis Howden \& Young, 1981

Eurysternini Vulcano, Martínez \& Pereira, 1960

Eurysternon foedus Guérin, 1844

Eurysternus plebejus Harold, 1880

Phanaeini Kolbe, 1905

Coprophanaeus edmonsi Arnaud, 1997

Coprophanaeus (Coprophanaeus) morenoi Arnaud, 1982

Coprophanaeus ohausi (Felsche, 1911)

Coprophanaeus(Coprophanaeus) corythus (Harold, 1863)

Oxysternon conspicillatum (Weber, 1801)

Oxysternon silenus (Castelnau, 1840)

Phanaeus pyrois Bates, 1887

Sulcophanaeus noctis (Bates, 1887)

Canthonini Péringuey, 1901

Canthon aequinoctialis Harold, 1868

Canthon cyanellus LeConte, 1859

Canthon moniliatus Bates, 1887

Canthon septemmaculatus (Latreille, 1811)

Deltochilum gibbosum panamensis Howden, 1966

Deltochilum parileBates, 1887

Deltochilum psedoparile Paulina, 1838

Deltochilum violetae (Martínez, 1991)

Dichotomius gamboaensis Howden \& Young, 1981

Dichotomius globulus (Felsche, 1901)

Dichotomius horridus (Felsche, 1911)
Dichotomius satanas (Harold, 1867)

Cryptocanthon sp.Malagoniella astyanax (Olivier, 1789)

Onthophagini Lacordaire, 1856

Onthopahus sp.Onthophagus acuminatus Harold, 1880

Onthophagus praecellens Bates, 1887

Passalidae Leach, 1815

Passalinae Gravely, 1918

Passalini Reyes-Castillo, 1970

Passalus (Mitrarhinus) arrowi Hinchs, 1934

Passalus (Passalus) alticola (Kirsch, 1885)

Passalus (Passalus) interruptus (Linnaeus, 1758)

Passalus (Passalus) interstitialis Eschscholtz, 1829

Passalus (Passalus) punctiger Lepeletiel at Serville, 1825

Passalus (Passalus) spinulosus Hincks, 1934

Passalus (Pertinax) incertus Percheron, 1847

Paxilus leachi MacLeay, 1819

Proculinae Gravely, 1918Proculini Kaup, 1868

Odontotaenius striatopunctatus (Percheron, 1835)

Petrejoides subrecticornis Kuwert, 1897

Pseudoarrox caldesi Reyes-Castillo \& Pardo-

LocarnoVerres sternbergianus Zangi, 1905

Veturius platyrhinus (Westwood, 1845)

Verres onorei Boucher \& Pardo-Locarno, 1997

Trogidae MacLeay, 1819

Omorgus (Omorgus) suberosus (Fabricius, 1775)

Hybosoridae Erichson, 1847

Anainae Nikolajev, 1996

Anaides aff. planus Ocampo, 2006

Ceratocanthinae Martínez, 1968

Germarostes sp.

Geotrupidae Latreille, 1802

Geotrupinae Latreille, 1802

Atheyreini Howden y Martínez, 1963

Neoathyreus lanei (Martínez, 1952) 


\section{Apéndice II}

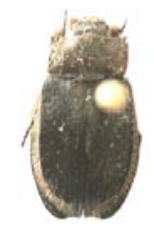

8
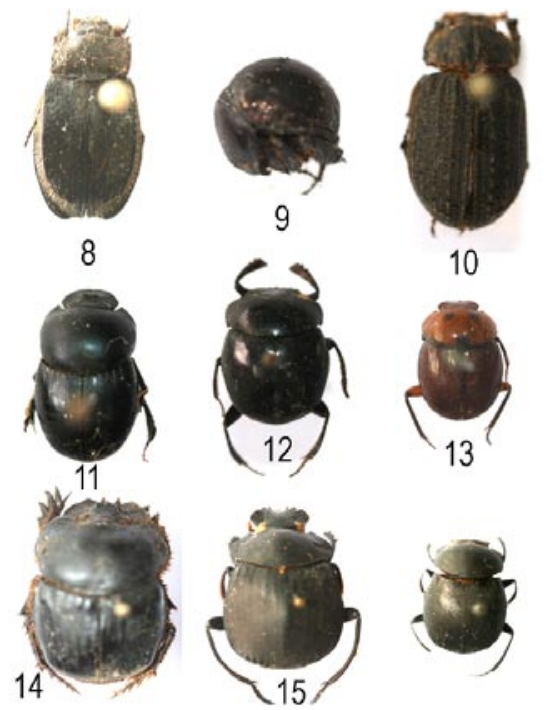

10
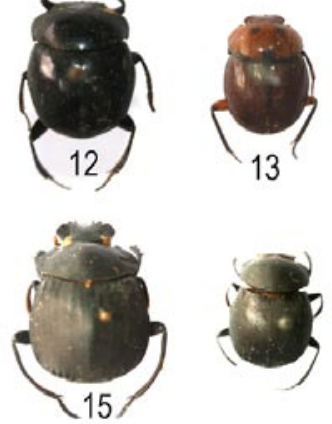

(8) Anaides aff. planus Ocampo; (9) Germarostes sp.; (10) Omorgus (Omorgus) suberosus (Fabricius); (11) Canthidium haroldi Preudhomme; (12) Canthon aequinoctialis Harold; (13) Canthon moniliatus Bates, (14) Coprophanaeus (Copro-phanaeus) corythus (Harold, 1863); (15) Deltochilum loperae González y Molano. (16) Deltochilum parile Bates.

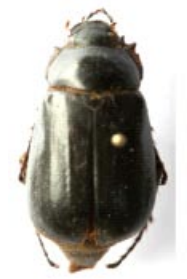

26
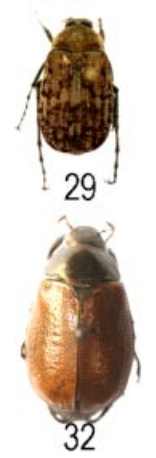

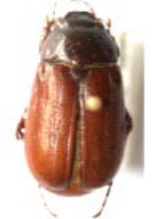

27

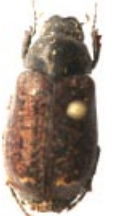

30

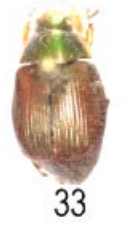

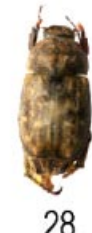

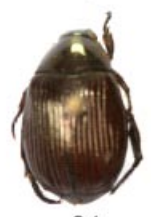

31

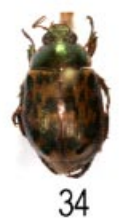

(26) Phyllophaga sp.1; (27) Phyllophaga sp.1; (28) Baribas sp.; (29) Isonychus sp.; (30) Plectris sp.1; (31) Paranomala cuacana (Ohaus); (32) Paranomala cupricollis (Chevrolat); (33) Paranomala medellina (Ohaus); (34) Paranomala undulata (Melsheimer).
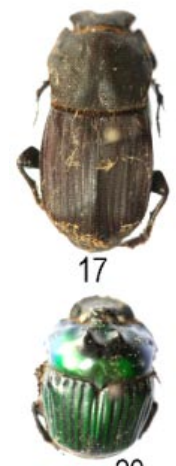

20

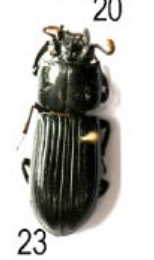

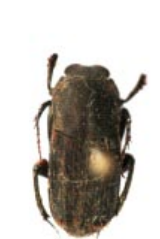

18
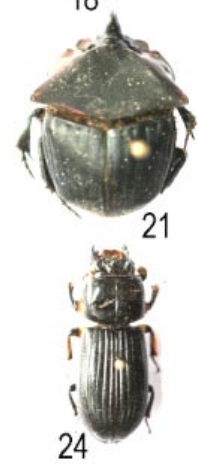

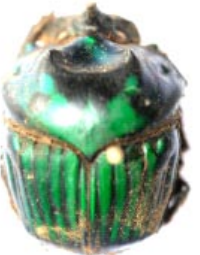

19
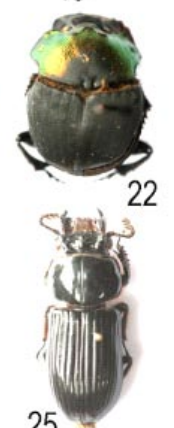

17) Eurysternon foedus Guérin; (18) Eurysternus plebejus Harold; (19) Oxysternon conspicillatum (Weber); (20) Oxysternon silenus (Castelnau); (21) Phanaeus pyrois Bates; (22) Sulcophanaeus noctis (Bates); (23) Passalus (Passalus) interruptus (Linnaeus); (24) Passalus (Passalus) spinulosus Hincks; (25) Veturius platyrhinus (Westwood).

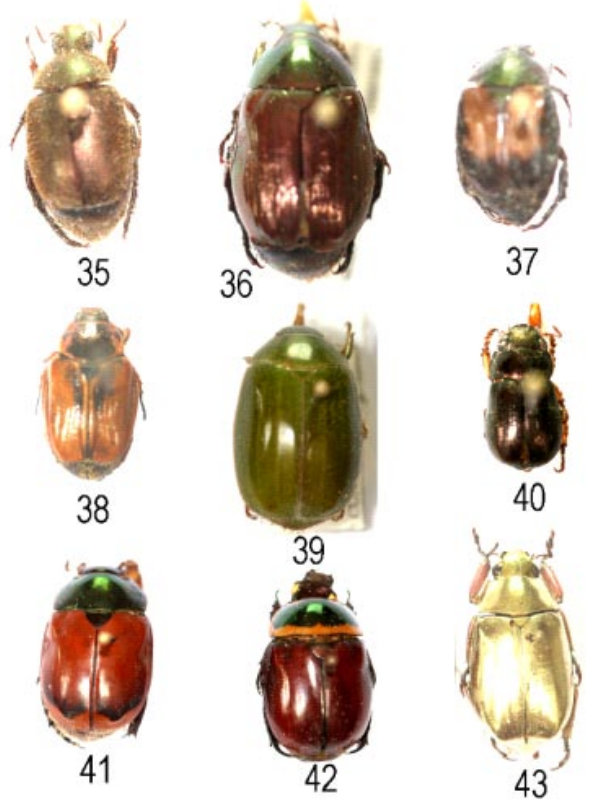

(35) Paranomala sp.1; (36) Paranomala sp.2; (37) Paranomala sp.3; (39) Strigoderma sp.1; (40) Platycoelia occidentalis Burmeister; (41) Leucothyreus femoratus Burmeister; (41) Chlorota jamesonae Soula; (42) Chlorota sp.1; (43) Chrysina argenteola (Bates). 


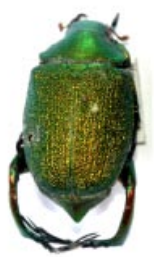

44
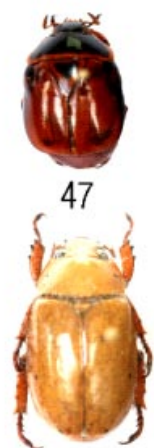

50

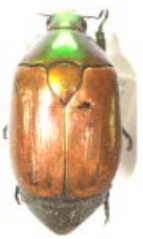

45

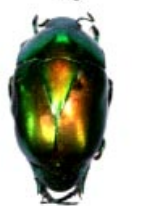

48

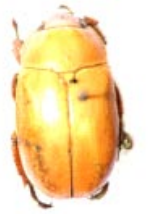

51

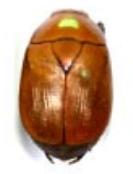

46
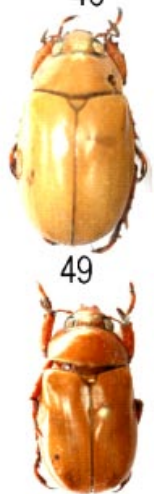

52
(44) Chrysophora chrysochlora (Latreille); (45) Dorystethus maximus Ohaus; (46) Dorystethus sp.; (47) Lagochile sp.1; (48) Macraspis lucida (Linnaeus); (49) Pelidnota chiriquina Bates; (50) Pelidnota notata Blanchard; (51) Pelidnota polita (Latreille); (52) Pelidnota prolixa Sharp.

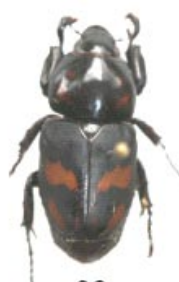

62

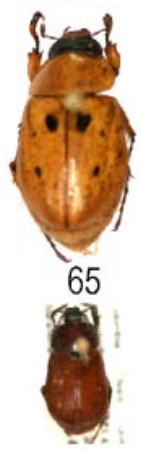

68

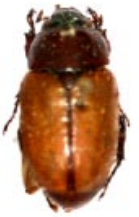

63
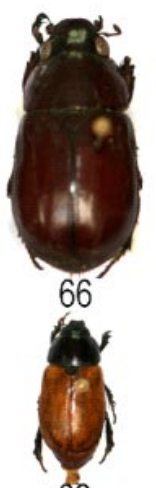

68

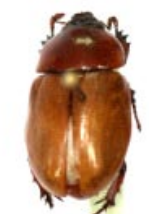

64

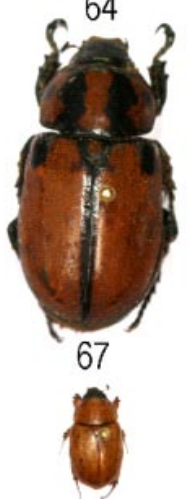

69
(62) Cyclocephala brevipennis Endrödi; (63) Cyclocephala gravis Bates; (64) Cyclocephala hiekei Endrödi; (65) Cyclocephala krombeini Endrödi; (66) Cyclocephala ligirina Bates, 1888; (67) Cyclocephala maffafa Burmeister; (68) Cyclocephala macrophylla Erichson; (68) Cyclocephala melanocephala (Fabricius); (69) Cyclocephala ovolum Bates.

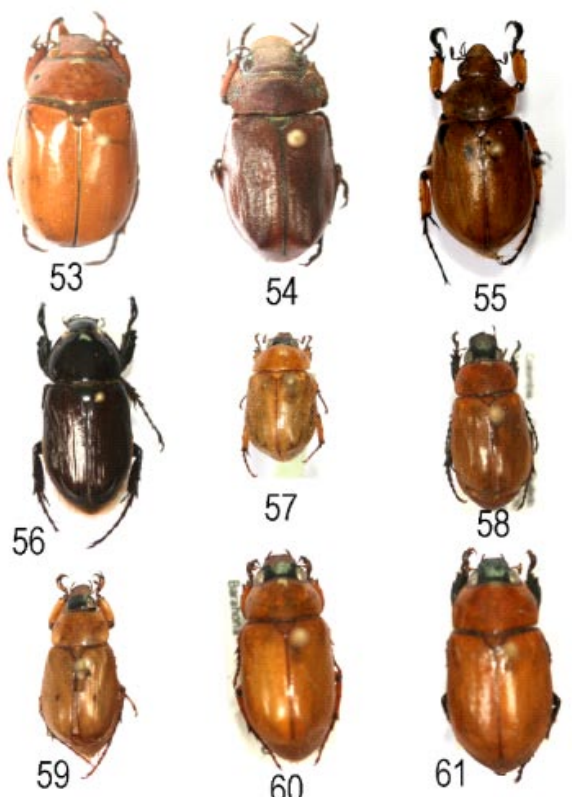

(53) Pelidnota prolixa Sharp; (54) Spodochlamys sp.; (55) Ancognatha vulgaris Arrow; (56) Aspidolea fuliginea Burmeister; (57) Aspidolea kuntzeni Höhne; (58) Aspidolea singularis Bates; (59) Cyclocephala amazona (Linnaeus); (60) Cyclocephala amblyopsis Bates; (61) Cyclocephala atripes Bates.

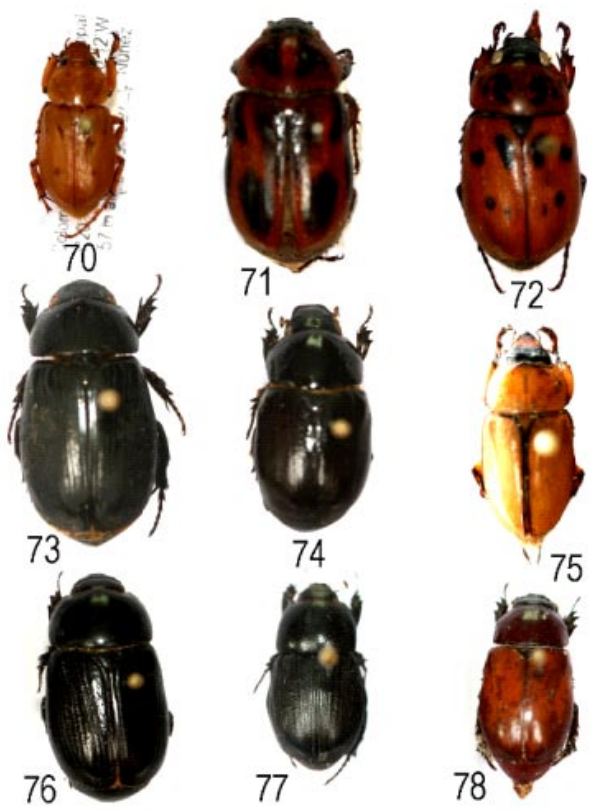

(70) Cyclocephala santaritae Ratcliffe; (71) Cyclocephala tutilina Burmeister; (72) Cyclocephala vidanoi Dechambre; (73) Dyscinetus dubius (Olivier); (74) Erioscelis columbica Endrödi; (75) Mimeoma acuta Arrow; (76) Stenocrates bicarinatus Robinson; (77) Euetheola bidentata (Burmeister); (78) Parapucaya mazonica Prell. 


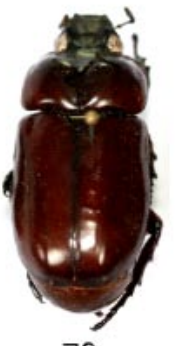

79

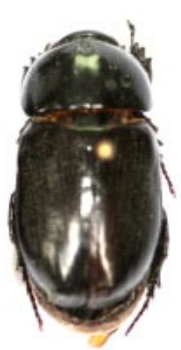

82
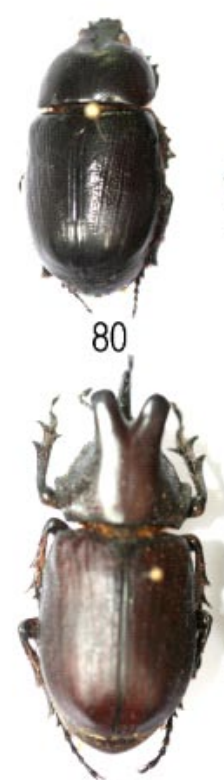

83

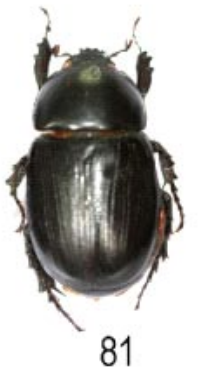

81

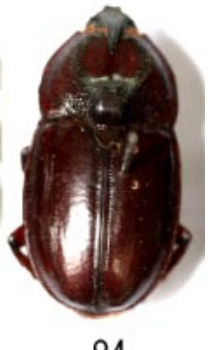

84
(79) Pucaya castanea Ohaus; (80) Tomarus ebenus(De Geer); (81) Tomarus fossor (Latreille); (82) Tomarus simillus (Endrödi); (83) Coelosis biloba (Linnaeus); (84) Enema pan (Fabricius).
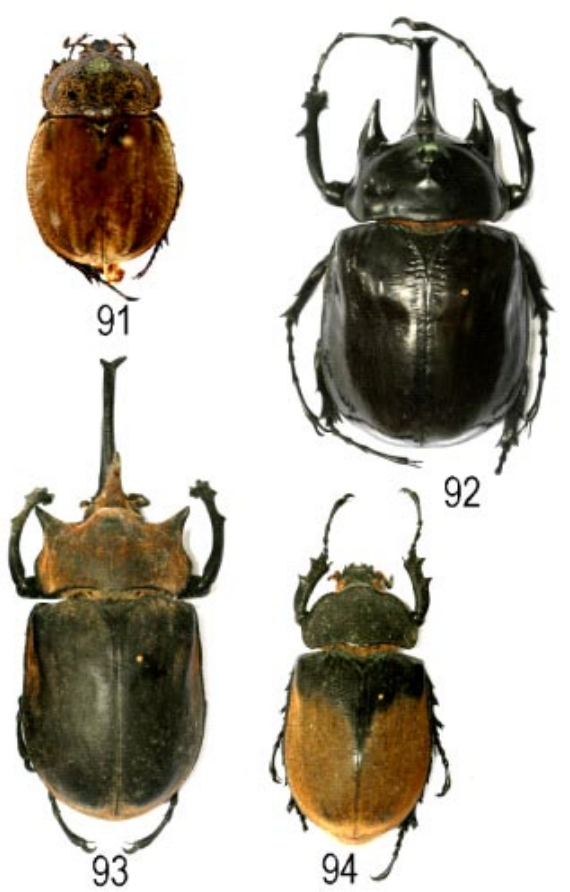

(91) Spodistes grandis Sternberg; (92) Megosoma acteon (Linnaeus); (93) Megasoma elephas (Fabricius)-Macho; (94) Megasoma elephas (Fabricius)-Hembra.

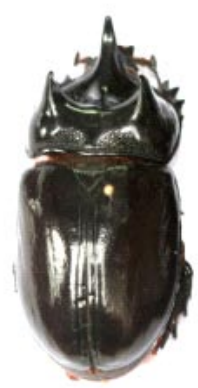

85

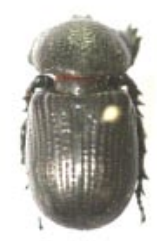

88

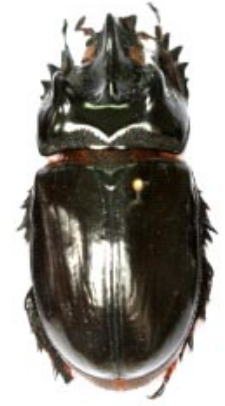

86

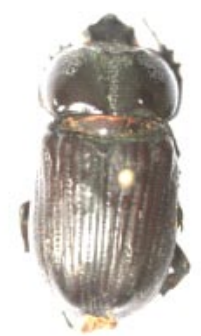

89

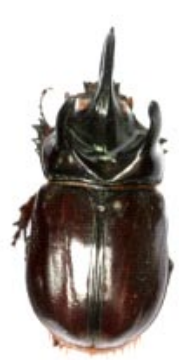

87

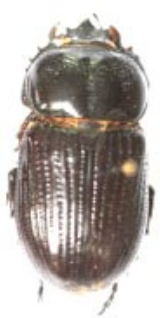

90
(85) Strategus aloeus (Linnaeus); (86) Strategus hipposederus Ratcliffe; (87) Strategus jugurta Burmeister; (88) Hemiphilerus elbitae Neita y Ratcliffe, 2010; (89) Homophileurus quadripuntactus (Beauve et Palisot); (90) Homophileurus tricuspis Prell.

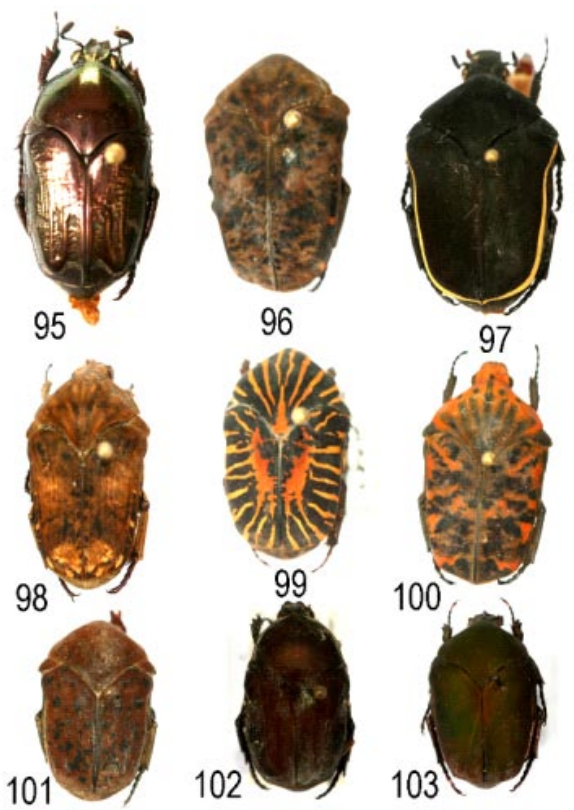

(95) Amithao decemguttatum (Waterhouse); (96) Gymnetis coturnix (Burmeister); (97) Gymnetis holosericea (Voet); (98) Gymnetis pardalis (Gory \& Percheron); (99) Gymnetis stellata (Latreille); (100) Gymnetis vandepolli (Bates); (101) Gymentis wallastoni Schaum; (102) Guatemalica hueti (Chevrolat); (103) Cotinis lebasi (Gory \& Percheron). 